\section{Cold Storage}

Cryopreservation techniques include the freezing, storing, and thawing of cells, tissues, or whole organisms so that the end result is viable and resembles the starting material. The motivation may be to maintain a type culture collection for research to ensure availability for reproductive or therapeutic use, or to establish a resource of organisms that are threatened by extinction. In general, liquid nitrogen temperatureapproximately $-130^{\circ} \mathrm{C}$, the temperature at which frozen water no longer sublimates and recrystallizesis used for such long-term storage. But, in order for cryopreservation to succeed, biologic material requires cryoprotectant agents to prevent damage that can occur from osmotic stress or ice crystal formation during freezing and/or thawing.

\section{Insurance Policy}

Mary Hagedorn, senior scientist at the Smithsonian National Zoologic Park in Washington, DC, whose duty station is the Hawaii Institute of Marine Biology, is investigating the use of cryopreservation with aquatic species. She has applied fertility techniques to the preservation of marine fish species, but also has realized the great need to preserve coral species. "We will lose all of our coral reefs due to global warming," she says. "Global warming is causing huge problems for corals, especially warm water corals in tropical reefs that live at the top of their physiologic range." Another threat is acidification of the oceans caused by an increase in dissolved carbon dioxide, which affects the ability of corals and other marine life to extract the calcium carbonate they need for shells, bones, and other structures. "We could see all ocean creatures disappear in 50 years, except jellyfish."

"Most of what people do to save these organisms," Hagedorn says, "is to restore and protect [habitat] areas.

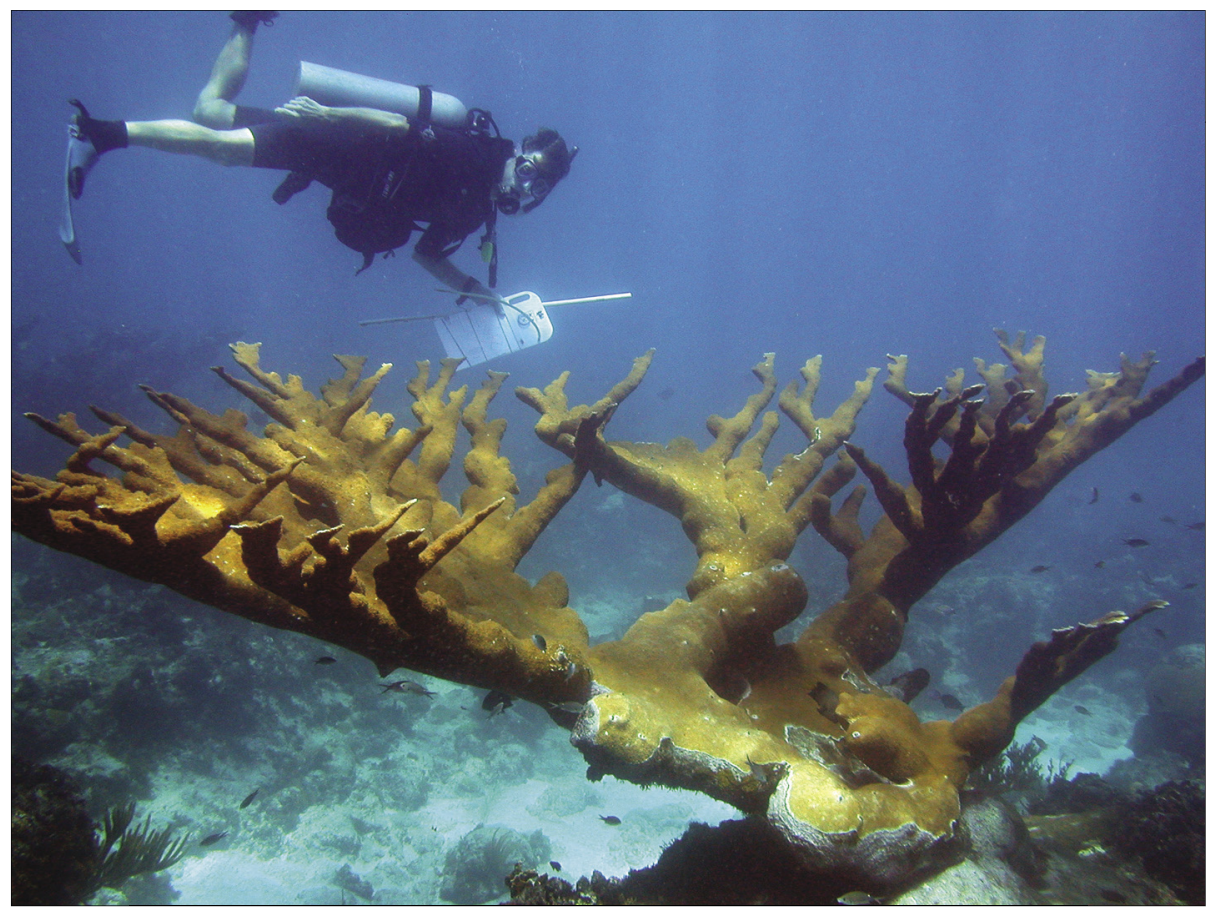

Elkhorn coral, Acropora palmata. Courtesy of Eric Borneman, University of Houston, Houston, TX.

This is great, but other actions are needed." Hagedorn is developing cryopreservation methods that could be used to reseed coral reefs, since coral sperm can be frozen and used to fertilize fresh oocytes. "I see myself and my science as an insurance broker for the future. I hope it's something we never have to use." Hagedorn has investigated freezing coral larvae, which she says is as challenging as freezing human oocytes, due to their size and fat content. Additionally, work on reproductive cells-and therefore larvae-is hampered by the animals' short reproductive season. But, she notes that much work on the physiology of these invertebrates remains to be done. "Where we are going next is really exciting," she says. "We are taking chips of polyps and freezing them, so we won't be tied to the breeding season." She hopes that by training people to collect polyps and freeze 50 to 60 pieces for each species, coral reefs could be reseeded in the future if necessary.

\section{"I see myself and my science as an insurance broker for the future."}

Hagedorn's group is also working on isolating coral stem cells. This would allow researchers to study coral diseases in vitro, which currently is not possible. One of the species she is working with is Elkhorn coral, Acropora palmata, one of the three most important reef-building species in the Caribbean, and which is a threatened species under the Endangered Species Act, according to the United States Fish and Wildlife Service. Hagedorn notes that one problem with this species' population in the Florida Keys is that, due to such low diversity, it consists almost entirely of clones. She would like to be able to introduce more diversity into this population by hybridizing it with material from related populations, and holds out hope that 
the Fish and Wildlife Service will allow her to do this.

\section{Empirical Methods}

Replacing coral reefs takes more than repopulating coral polyps, notes Jerry Brand, a professor in the School of Biological Sciences at the University of Texas in Austin, Texas. The coral community relies on zooxanthellae, symbiotic dinoflagellates (algae) that provide corals with energy. Although Brand does not work with zooxanthellae, his work with cryopreservation of other algae species is applicable to many microorganisms and other species. Algae, Brand notes, make up an important component of many ecosystems, and if coral reefs are to be replaced, the entire ecosystem may need to be restored.

An advantage of maintaining frozen stocks of microorganisms is the ability to preserve strains whose precise identity is known. Continually passaged strains under the selective pressure of culture are likely to develop genetic or epigenetic changes that may only be advantageous in vitro, leading to the loss of the original, well-characterized strain. In addition, maintaining cultures in vitro is resource-, time-, and laborconsuming. Brand describes the algae collection at the University of Texas as one of the largest and most diverse in the world, containing about 400 strains. About two-thirds of these are cryopreserved. Brand notes that most fresh water strains, including those that exist in soil or as epiphytes, those that live in fresh water, and those that are less than 40 micrometers in diameter, can be frozen. Species that are difficult to cryopreserve are marine species, have large cells, are multicellular, or have large vacuoles.

Brand says that much of the work in his field has been empirical. For species that are easy to freeze, almost any freezing protocol works, and for those that are finicky, nothing works. The right cryoprotective agent is important, along with an appropriate cooling rate. If freezing is too rapid, damaging ice crystals can form, and if freezing is too slow, osmotic changes can crush cells. Cytoskeletal damage and oxidative damage during freezing or thawing may also contribute to a lack of viability. One issue of particular interest is why multicellular algae are so difficult to preserve. Chlamydomonas, a small, unicellular, flagellate green alga, is easy to cyropreserve. Gonium, another flagellate green alga, often existing in 4-cell colonies, can also easily be frozen. For larger colonies, such as Pandorina, which exists in colonies of 8,16 , and occasionally more cells can be successfully cryopreserved as well. The limit appears to be reached with organisms like Volvox, a species that exists in spherical colonies of $2^{n}$ cells, usually 512 or 1024 cells. Brand hypothesizes that intracellular communication in the multicellular forms, which signal damage in one cell to the rest of the colony, may be responsible for a cryopreservation size limit of about 32-64 cells in algae.

Brand's group is trying different protocols, including different combinations of cryoprotectants, different freezing and thawing protocols, and varying culture densities, but their main effort is to maintain their collection. The three most common cryoprotectants for algae are methanol, particularly for fresh water species, dimethyl sulfoxide (DMSO) in particular for marine algae, and glycerol for cyanobacteria.

\section{Banking on It}

Better cryoprotectants are critical to the work being done by Jason Acker at Canadian Blood Services in Edmonton, Alberta, Canada, who is researching methods for freezing red blood cells (RBCs) for transfusion. "The amazing thing is that we are still using old technologies [such as the use of glycerol and DMSO] developed in the 1950s," he says. "It's to our detriment that the early technology did work." Now, this technology is cumbersome and expensive, and used mainly for extremely rare blood types and by the military. Although glycerol and DMSO have been used to cryopreserve human RBCs and stem cells, respectively, they do have drawbacks: there was discussion after September 11, 2001 about cryopreserving large stockpiles of type 0 negative RBCs (the socalled universal donor type), but the technology to deglycerolize RBCs after thawing is too time-consuming to meet the demand for blood during an emergency. So, a key research goal is to find a cryoprotectant that could allow transfusion directly from freezer to patient.

\section{"The amazing thing is that we are still using old tech- nologies developed in the 1950s."}

Acker says that researchers are looking at natural systems to determine how organisms in nature survive freezing. One natural cryoprotectant that has been identified is the sugar trehalose, which is currently used in the pharmaceutical industry to preserve proteins. It exerts its effects intracellularly within organisms that use it for natural cryoprotection, but it is not permeable across RBC membranes, so a method for introducing it into cells is needed. Also, Acker notes, trehalose and other cryoprotectant sugars probably do not function in living organisms in isolation. He observes that a good endpoint is still needed for the function of transfused RBCs, either stored at refrigerator temperatures, or after freezing and thawing. He'd like to see more focus on membrane changes, including surface molecule expression, and oxidative changes.

A pressing need, Acker says, is to get researchers like him collaborating 
with blood banking and stem-cell banking personnel in order to share expertise, identify available resources, and pinpoint technology gaps. Blood banks are struggling with the daily problems of delivering products. Acker sees his work as a bridge between research and practice that can foster communication about new technologies, and identify and develop appropriate solutions to problems.

\section{Crop Conservation}

The Global Crop Diversity Trust shares Hagedorn's concerns about losing species diversity. Charlotte Lusty, a scientist with the Trust, notes that one problem with banks of seed crops is that all seeds need to be regenerated to avoid loss of viability over time. As with algae, this is expensive and time-consuming, and some seed crops, such as coconut, are difficult to maintain and ship. Although the Global Seed Vault near Longyearbyen, Norway archives millions of seeds, some crops will not survive cold storage, and will need to be cryopreserved at lower temperatures. The Trust is trying to determine the best cryopreservation techniques for particularly important and difficult-topreserve food crops like yam, cassava, maize, banana, breadfruit, coconut, potato, and sweet potato.

-Lynne Lederman is a freelance medical writer in Mamaroneck, NY. 